\title{
Análisis del interés cognoscitivo en clases de matemáticas
}

\author{
Analysis of cognitive interest in math classes
}

Yolanda Rosas-Rivera ${ }^{1}$

\section{RESUMEN}

El aprendizaje de las matemáticas está relacionado con factores cognitivos $\mathrm{y}$ emocionales. Desde el enfoque histórico cultural es posible analizar ambos factores mediante el estudio de los intereses cognoscitivos para que el maestro pueda organizar sus métodos de enseñanza dirigidos hacia el desarrollo tanto del pensamiento teórico como de la personalidad (actitud creativa). El objetivo de la presente investigación es identificar y describir el interés cognoscitivo que desarrollan los alumnos durante las clases de matemáticas. Se presentan las experiencias escolares de dos grupos de tercer grado de primaria de colegios privados que identifican un método de enseñanza propio para la enseñanza de las matemáticas. Se concluye que el interés cognoscitivo se desarrolla cuando las actividades incluyen conceptos matemáticos, uso de materiales y solución en grupo.

Palabras-clave: Motivación escolar. Conceptos matemáticos. Método de enseñanza. Personalidad infantil.

\begin{abstract}
Learning mathematics is related to cognitive and emotional factors. From the historicalcultural approach, it is possible to analyze both factors by studying cognitive interests so that the teacher can organize his teaching methods aimed at the development of both theoretical thought and personality (creative attitude). The objective of this research is to identify and describe the cognitive interest that students develop during math classes. The school experiences of two groups of third grade of primary from private schools that identify their own teaching method for the teaching of mathematics are presented. It is concluded that the cognitive interest develops when the activities include mathematical concepts, use of materials and group solution.
\end{abstract}

Keywords: Learning motivation. Math concepts. Teaching method. Childhood personality.

1 Doctorado Interinstitucional en Educación, Universidad Iberoamericana de Puebla, México. Profesora en Centro Universitario de la Costa, Universidad de Guadalajara. ORCID: https://orcid.org/0000-0002-0422-7128. E-mail: yolanda.rosas@academicos.udg.mx. 


\section{RESUMO}

Aprender matemática está relacionado a fatores cognitivos e emocionais. A partir da abordagem histórico-cultural, é possível analisar ambos os fatores estudando os interesses cognitivos para que o professor organize seus métodos de ensino voltados ao desenvolvimento do pensamento teórico e da personalidade (atitude criativa). $\mathrm{O}$ objetivo desta pesquisa é identificar e descrever o interesse cognitivo que os alunos desenvolvem durante as aulas de matemática. São apresentadas as experiências experiências escolares de duas turmas do terceiro ano do ensino fundamental de escolas particulares que identificam seu próprio método de ensino para o ensino de matemática. Conclui-se que o interesse cognitivo se desenvolve quando as atividades incluem conceitos matemáticos, uso de materiais e solução em grupo.

Palavras-chave: Motivação escolar. Conceitos matemáticos. Método de ensino. Personalidade infantil.

\section{Introducción}

Los planes y programas oficiales en México consideran que la principal razón para el aprendizaje es relacionar los contenidos revisados en la escuela con el contexto del alumno, ya que dicha vinculación dará sentido al aprendizaje de cada alumno. Por lo que una de las tareas más relevantes del maestro señalado en el plan de estudios es "mantener y promover el interés y motivación por aprender" (SEP, 2017 p. 73). Para lo cual se propone que el maestro utilice el aprendizaje basado en preguntas, problemas y proyectos. Sin embargo, solo se simplifica a considerar el interés del alumno a partir de identificar lo que le gusta, el contenido de sus vivencias cotidianas y atender a sus preguntas, las cuales están dirigidas hacia los procedimientos (WEISS; BLOCK; CIVERA; DAÁVALOS; NARANJO, 2019; ARÉVALO, 2015).

En la investigación de Padilla y Mejía (2020) se refiere a la motivación como el impulso que guía, orienta, intensifica y mantiene la conducta ante alguna situación determinada con la idea de obtener algún resultado propuesto. Esta motivación puede ser expresada por los alumnos de formas diversas, por ejemplo en la búsqueda para aprender algún tema, obtener una buena calificación, involucrarse o evitar alguna actividad, sentirse capaz o incapaz de realizar una actividad. Estos autores aplicaron una encuesta a alumnos mexicanos de quinto y sexto de primaria para identificar cuáles eran los factores motivacionales con mayor intensidad que se relacionan con el aprendizaje. Los 
resultados mostraron que el esfuerzo (repaso de tareas), el interés (captación de la atención) y la autoeficacia (sentirse capaz para realizar una actividad) se correlacionan de forma positiva con el rendimiento académico. A pesar de ser un estudio cuantitativo es posible considerar que el interés obtiene un papel importante dentro de la motivación escolar.

En otro estudio pero desde la perspectiva Vygotskiana, Sánchez y Dupico (2006) consideran que los motivos escolares no puede estudiarse como elementos aislados, sino que debe considerarse como formaciones que incluyen las necesidades, ideales, aspiraciones y convicciones, lo que conlleva a la unidad entre lo cognitivo y lo afectivo emocional. Específicamente, los autores enfatizan que los intereses pueden comprenderse como manifestaciones emocionales de las necesidades cognitivas de los alumnos. Además, proponen en su investigación el uso de la encuesta, observación de clases y la composición para identificar los intereses que tienen los alumnos de quinto y duodécimo grado hacia las clases de educación física, también integran en su análisis a los maestros de grupo. Este estudio aunque es una propuesta metodológica permite retomar algunos indicadores para caracterizar los intereses que participan en la actividad de estudio, considerando las investigaciones de Schukina (1968). Por ejemplo, los autores proponen identificar la profundidad del interés (curiosidad, teórico, cognoscitivo), y si se satisface o se mantiene en expectativa durante las clases.

Continuando con la perspectiva del enfoque de Vygotski (2006), los intereses relacionados con el aprendizaje son desarrollados dentro del contexto escolar y en interacción con los maestros y compañeros, aunque es el maestro quién tiene la posibilidad de organizar el contenido para que dicho interés se centre en el aprendizaje escolar y no solo en aspectos secundarios (aprobación del maestro o de sus padres, juego o en la calificación). Consideramos que es posible cumplir ese objetivo de desarrollo si el docente reflexiona sobre aquellas condiciones de su práctica que favorecen al interés cognoscitivo, de qué forma es posible presentar los contenidos académicos (conceptos, acciones) y en qué momento del desarrollo presentarlos. Como hemos enfatizado en otras investigaciones (ROSAS, 2019; ROSAS; SOLOVIEVA, 2019) la tarea del docente 
requiere de conocimientos del desarrollo psicológico, propios de la asignatura a enseñar y de la pedagogía para poder organizar los métodos de enseñanza y tener un impacto positivo en el desarrollo de los alumnos.

Una de las asignaturas con menor comprensión y reflexión, además de importantes dificultades en su aprendizaje para los alumnos y en su enseñanza para los docentes, son las matemáticas en la educación primaria (WEISS; BLOCK; CIVERA; DÁVALOS; NARANJO, 2019). Es conocido el panorama de los resultados de evaluaciones nacionales e internacionales de los alumnos de tercer grado de primaria, y a pesar de que existen estudios con estrategias específicas para mejorar esos puntajes y el aprendizaje de los alumnos, aún no se logra una coherencia teórica metodológica que oriente a los docentes y alumnos (ROSAS; SOLOVIEVA, 2019).

La enseñanza- aprendizaje de las matemáticas puede ser estudiada desde la perspectiva centrada en el alumno, en el maestro o en la interacción de ambos. Nosotros hemos considerado, desde la teoría de la actividad, que tanto el docente como alumno se encuentran en la actividad de estudio, por lo que se requiere estudiar la enseñanza-aprendizaje como un proceso en el que participan de forma activa, creativa y transformadora los docentes y alumnos (SOLOVIEVA; GONZÁLEZ; ROSAS; MATA; MORALES, 2020). En investigaciones anteriores (ROSAS; SOLOVIEVA; QUINTANAR, 2017; ROSAS, 2019) hemos estudiado el desarrollo de los conceptos matemáticos a partir de métodos de enseñanza organizados y dirigidos hacia el pensamiento teórico. Sin embargo, aunque es posible identificar la participación de los motivos escolares y de la personalidad en nuestros trabajos previos, consideramos necesario estudiar de forma específica los intereses cognoscitivos que se desarrollan durante las clases de matemáticas.

De esta forma nos propusimos indagar en las condiciones de la enseñanza y aprendizaje de las matemáticas que favorecen el desarrollo del interés cognoscitivo de los alumnos y maestros de educación primaria de dos colegios privados, que cuentan con un método específico de enseñanza de las matemáticas. Nos planteamos las siguientes preguntas como orientaciones para nuestro trabajo: ¿Cómo se desarrolla el interés cognoscitivo en las clases de matemáticas? Además, al existir diversas formas de enseñar matemáticas y sin 
una propuesta clara en los programas oficiales de enseñanza o en los libros de textos como lo refiere Weiss, Block, Civera, Dávalos y Naranjo (2019), nos propusimos identificar las diferencias del contenido científico de la propia matemática que conocen las maestras, de esta manera nos preguntamos ¿qué contenido matemático favorece la aparición del interés cognoscitivo?

Para cumplir nuestro objetivo organizamos la información de la siguiente forma, primero describimos qué se puede entender por interés cognoscitivo y su desarrollo durante la edad escolar. En un segundo apartado se presenta los conceptos y habilidades matemáticas que son posible enseñar en la escuela primaria. En tercer lugar, proponemos un análisis de dos experiencias de maestras mediante la descripción de los métodos de enseñanza de las matemáticas que utilizan, un método fue abordado desde el modelo constructivista, y el otro desde la teoría de la actividad. Finalmente, identificamos algunas actividades y orientaciones que favorecen el desarrollo del interés cognoscitivo, las cuales surgen del análisis de nuestros trabajos de formación y evaluación dinámica de conceptos matemáticos en alumnos de primaria.

\section{La actividad de estudio e interés cognoscitivos desde la teoría de la actividad}

Vygotsky (1995) investigó el uso y el lugar de los principios psicológicos en el campo pedagógico. También, planteó que el ser humano se desarrolla a partir de la apropiación de instrumentos (físicos como herramientas y psicológicos como los signos). Dicho proceso de apropiación siempre es de origen sociocultural y se realiza en forma de diversas actividades prácticas y relaciones sociales.

Por primera vez en la Psicología, Vygotsky (1984) menciona que las actividades educativas pueden ser favorables o desfavorables para el proceso de adquisición de conceptos. Profundizando esta idea, los seguidores de Vygotsky (DAVÍDOV, 1988; TALIZINA, 2019; GALPERIN, 2009), han estudiado los efectos de diversos métodos de enseñanza sobre los sistemas de conceptos que se forman en los alumnos. Desde el tiempo de Vygotsky (1984), se inició el estudio de las condiciones favorables que permitan garantizar mejor el nivel de adquisición de 
sistemas de conceptos, entre otras áreas, de los conceptos matemáticos.

El ingreso del niño a la escuela primaria debe marcar una diferencia en el desarrollo psicológico del niño y por consecuencia una nueva organización de actividades, debido a que tanto la posición social como la actividad de aprendizaje sufren un cambio (DAVIDOV; MAKOROVA, 1987; BOZHOVICH, 1976). Esto conlleva a pasar de una actividad rectora de desarrollo del juego temático de roles (preescolar) hacia la actividad de estudio, siguiendo la propuesta de periodización de la teoría de la actividad (ELKONIN, 2011).

La actividad de estudio permite que el alumno asimile la relación teórica de la realidad, la base del pensamiento teórico, formaciones como el carácter voluntario de las acciones, la imaginación y la actitud creativa (DAVIDOV; MAKOROVA, 1987). De acuerdo con Davidov (1988), la enseñanza puede desarrollar la posición vital activa, es decir, la necesidad de crear, lo que es posible considerar como un indicador del desarrollo de la personalidad.

\subsection{El desarrollo de la personalidad en la edad escolar}

La formación de la personalidad del niño transcurre por diferentes vías en dependencia, una de ellas es la preparación para el aprendizaje escolar y la otra es el sistema de influencias pedagógicas que recibe (BOZHOVICH, 1987). Las investigaciones de la autora muestran que los alumnos ingresan a la escuela con el agrado por estudiar y se sienten atraídos por los trabajos serios. En la actitud que expresen los alumnos es posible identificar sus intereses cognoscitivos. Los alumnos de los primeros grados expresan un sentido social que se refleja en la importancia de las notas para ellos y en la aprobación de los maestros. Posteriormente, los niños se interesan por aquellas tareas que les generan una tensión intelectual, prefieren tareas difíciles que fáciles. Sin embargo, estos intereses pueden no ser estables o suficientes para contribuir al aprendizaje en los grados posteriores, la autora refiere que existe un cambio negativo. Los alumnos muestran un comportamiento de cansancio hacia las obligaciones escolares, su disciplina disminuye y la autoridad del maestro decae. Las fallas en la organización del proceso docente influyen en esa regresión, por ejemplo, los errores 
de contenido y organización de la enseñanza escolar primaria (DAVIDOV, 1988). Debido a que el interés cognoscitivo es una necesidad general y esencial y su no satisfacción podría ser la causa de esa regresión.

La hipótesis del análisis pedagógico conlleva a identificar cómo los docentes enseñan las materias en las escuelas para formar correctamente y de forma estable ese interés cognoscitivo, y que este se transforme en un motivo escolar interno. Bozhovich $(1987 ; 1976)$, Davidov y Makorova (1987) y Talizina (2019) describen que la mayoría de los contenidos revisados en las clases de primaria son concretos, sin una relación con la actividad intelectual (conceptual), con una cantidad innecesaria de ejercicios que solo obliga a los alumnos a memorizar de forma mecánica. Por lo que es posible relacionar dichos métodos con el insuficiente desarrollo de la personalidad de los alumnos, particularmente del motivo escolar e interés cognoscitivo.

Ante el panorama anterior, aceptando que existe un periodo critico donde el interés cognoscitivo no se satisface por los errores en la enseñanza, es necesario que los docentes preparen las condiciones para la formación de una motivación escolar a través de la organización de los conceptos científicos de cada materia. Las investigaciones de Davidov y Elkonin, citados por Talizina (2019), muestran que cuando los métodos de enseñanza consideran el desarrollo del pensamiento infantil y los conceptos científicos, los alumnos logran satisfacer su curiosidad e interesarse hacia lo que los rodea, tienen aspiraciones creadoras y mantienen un interés hacia el estudio.

Por último, Bozhovich (1987) refiere que conforme el alumno logra incluirse a esa nueva actividad de estudio y es mas independiente del adulto, surgen determinadas relaciones con sus compañeros. Los alumnos muestran interés por trabajar con sus compañeros, por jugar entre ellos y realizar en colectivo las tareas propuestas por el docente. Esto nos lleva a considerar también que las relaciones sociales entre los alumnos, las cuales por propuesta de Bozhovich (1976), también pueden ser organizados correctamente y tener un mejor impacto en las prácticas pedagógicas.

De esta manera podemos considerar que la enseñanza que conlleva al 
desarrollo en la escuela primaria debe incluir en su organización el análisis de cómo se relacionan los procesos cognitivos y afectivos durante la realización de las actividades escolares. Una propuesta para dicha relación es el trabajo con los intereses cognoscitivos, los cuales incluyen elementos intelectuales y emocionales.

\subsection{Intereses cognoscitivos}

El interés cognoscitivo está relacionado con los procesos intelectuales, emocionales y volitivos, es el motivo y fuerza motriz importante de la actividad. Este interés permite que la actividad intelectual sea reflexiva y analice los conocimientos de un problema escolar, además de que le permite adquirir un mayor carácter para ser productiva (SCHUKINA, 1965).

Las características del interés cognoscitivo son: 1) una actitud compleja hacia los objetos y fenómenos de la realidad que nos rodean, 2) carácter intelectual, está dirigido hacia la actividad cognoscitiva, es decir, hacia el dinamismo, la búsqueda de la esencia, las relaciones profundas y el dominio de las leyes que rigen el verdadero conocimiento, 3) carácter selectivo, lo cual se relaciona con aquello que necesita, con lo que para la propia personalidad tiene una especial importancia, dichas necesidades pueden estar establecidas por las edades psicológicas, 4) tiñe de emociones la actividad mental, el sentimiento de satisfacción y entusiasmo por el trabajo realizado provoca una participación más activa, las expresiones de sorpresa y admiración son ocasionadas por el conocimiento nuevo durante las clases, 5) intencionalidad volitiva, este tipo de interés es activo, posibilita al sujeto para tener una actividad productiva, es decir, el interés no puede quedarse solo en el pensamiento sino que debe orientar hacia la acción, a partir de la búsqueda activa y orientada de los procedimientos para resolver las tareas cognitivas, debido a que el sujeto percibe el deseo de esforzarse para concluir exitosamente las tareas (SCHUKINA, 1965).

Las fases de desarrollo del interés cognoscitivo permiten identificar en qué proceso se encuentra la actitud del alumno en alguna asignatura. En el estudio de Sánchez y Dopico (2006) utilizan las siguientes fases para estudiar los intereses cognoscitivos hacia la asignatura de educación física en alumnos de 
quinto y sexto grado. Por lo que es posible continuar el análisis de dichas fases, que son retomadas también de los estudios de Schukina (1965). La primera fase es la de curiosidad. La curiosidad es el tipo más elemental de orientación y se relaciona con la novedad del objeto, en el ámbito escolar el alumno puede solo atender al elemento divertido que le proporciona la asignatura. La siguiente fase es el afán de saber, el cual contiene el deseo de conocer los límites de ello invisible, el interés cognoscitivo se da a partir de las emociones de alegría y la admiración por saber, es posible identificar en el niño el cuestionamiento de saber porqué de los fenómenos y objetos que le rodean. Posteriormente, el interés cognoscitivo aparece como una intencionalidad especial de la personalidad por conocer la realidad, presenta un movimiento progresivo hacia el análisis profundo y amplitud de la esencia de los fenómenos. En este momento en el interés cognoscitivo se presenta una tensión mental, el esfuerzo volitivo que lo conduce a superar las dificultades y buscar activamente la solución. La última fase es el interés teórico, este implica no solo el deseo de saber sino de aplicar los conocimientos sobres leyes y fundamentos teóricos. Por lo que su principal característica es la acción activa sobre el mundo (SCHUKINA, 1965).

Para el desarrollo de los intereses cognoscitivos se requiere de un trabajo organizado y sistematizado, si bien las fases del interés cognoscitivo pueden presentarse en un mismo acto (SÁNCHEZ; DOPICO, 2006; DOMINGUEZ, 2015), es necesario que el docente analice hacia donde va dirigir a los alumnos para que estos logren un mejor desarrollo intelectual y por ende el de la personalidad. El maestro debe identificar las actividades propias de la asignatura que constituyan la fuente de creación y formación de los interés cognoscitivos de los alumnos.

Desde la propuesta de Schukina (1965), Davidov (1988) y Talizina (2019) la activad de estudio es la principal fuente de desarrollo de los intereses cognoscitivos. De forma específica Schukina (1965), Sánchez y Dopico (2006) y Dominguez (2015) refieren que el contenido del estudio es necesario porque está orientado hacia el conocimiento de objetos, fenómenos y procesos de la realidad. Entonces, durante clases el niño conoce esos objetos de la ciencia y puede generar cierto agrado de interés hacia estos. Una característica que han 
identificado los autores anteriores es la presentación de las relaciones entre las asignaturas, debido a que esto le permite conocer el mundo objetivo de forma amplia. Para lo cual Talizina (2019) también propone la presentación de sistemas de conceptos de una asignatura y la relación entre ellas, principalmente la formación de conceptos relativos y no solos los absolutos.

Otra característica de la actividad de estudio que favorece la formación del interés cognoscitivo es la formación de hábitos y capacidades. El estudio le permite al alumno utilizar los conocimientos que adquiere en la escuela en su vida diaria Schukina (1965), por ejemplo, en la escuela se le enseña a comprender lo que lee, a planificar y expresar sus ideas.

A partir del estudio de Schukina (1965) se han identificado que la enseñanza puede considerar algunas condiciones para favorecer el interés cognoscitivo. El primero que propone es crear una situación emocional, ya que estos conllevan al niño a tener un afán de saber algo nuevo, algo interesante que le invite a desarrollar su actividad intelectual. Dicha situación emocional no puede confundirse con las estrategias de animación o la actitud forzada del docente, sino debe generar una armonía, en relación con el interés cognoscitivo, entre los sentimientos del docente y del alumno, que ambos compartan el interés de saber. Algunos otros medios es la expresión de la "palabra emocional del docente" la cual se dirige hacia la descripción viva de los fenómenos, los hechos, los descubrimientos científicos, los personajes históricos. De esta condición se busca también que el alumno aprenda a valorar su esfuerzo, que no perciba pesado o aburrido su trabajo.

El desarrollo de la actividad intelectual le permite al alumno ser consciente y reflexivo sobre el éxito que logra durante las clases. Por lo que se considera también una condición en la enseñanza. La organización de la enseñanza tiene que plantear las tareas con el objetivo de que para solucionarlas los alumnos desarrollen habilidades generales del pensamiento (TALIZINA, 2019), por ejemplo la solución de problemas matemáticos debe ser dirigida hacia la solución de la pregunta final y no solo de la operación matemática (ROSAS; SOLOVIEVA, 2019). Así mismo, la enseñanza debe mostrar cómo solucionar esas tareas y dirigirlos no solo hacia la imitación o repetición de acciones sino llevarlos hacia la búsqueda de 
nuevas relaciones, es decir, la actividad creativa.

Lo anterior es posible lograr a partir de algunas tareas particulares, que propone (SCHUKINA, 1965): 1) el uso de preguntas que implican comparaciones, identificar diferencias o que permitan la explicación, así como que sean claras y con una entonación adecuada, 2) la diversidad en las tareas, tanto en complejidad como en cantidad, 3) generar actividades que dirijan a los alumnos hacia el uso de los conceptos y procedimientos aprendidos en el aula, es decir, que relacionen a la actividad intelectual con la actividad práctica, 4) el trabajo colectivo, analizar de forma colectiva dichos conocimientos. Así, el docente debe organizar cuidadosamente tanto los contenidos como los métodos de enseñanza y mantener en estado emocional la actividad intelectual.

Coincidiendo con la tarea de investigar el contenido del estudio que permita al alumno desarrollar la actividad cognoscitiva y su personalidad. En el siguiente apartado presentaremos el caso de la enseñanza de las matemáticas, describiremos el contenido científico que es necesario trabajar en las clases. Es posible que otros investigadores elijan otros enfoques de la psicología o la pedagogía o de la propia didáctica de la matemática, sin embargo el contenido de los conceptos matemáticos deben contener la misma esencia, sería ilógico pensar que el concepto de número dependa de la postura teórica, lo que si es posible que difieran son los elementos abordados, por ejemplo, en la mayoría de las investigaciones, la cantidad y la asociación de símbolos son los elementos más trabajados (ROSAS; SOLOVIEVA, 2019).

\section{La enseñanza de las matemáticas en la escuela primaria desde la teoría de la actividad}

Como hemos mencionado en el primer apartado y de acuerdo con Davídov (1988), los psicólogos y pedagogos deben considerar que el ingreso del alumno a la escuela se caracteriza por la formación de conceptos científicos, los cuales requieren de un procedimiento distinto e inesperado en comparación a como el niño aprendía en el preescolar. El principio es que la educación debe conllevar al desarrollo, por lo que los programas deben considerar acciones que influyan sobre este. 
Uno de los objetivos en los últimos años de la teoría de la actividad aplicada a la enseñanza (TALIZINA; SOLOVIEVA; QUINTANAR, 2010; SOLOVIEVA; GONZALEZ; ROSAS; MATA; MORALES, 2020) ha sido convertir el proceso no organizado y espontáneo de asimilación en un proceso dirigido y organizado, en el sentido de identificar aquellos conceptos que deben formarse. Además, debe tenerse en cuenta que en cualquier sistema para dirigir el proceso de asimilación se estructura sobre la base de determinada concepción psicológica sobre el proceso a dirigir. Antes de abordar el proceso de asimilación es necesario comprender el modelo desde el cual se organiza el contenido de los métodos, en caso contrario se puede cometer el error de trabajar con metodologías incorrectas y caer en los métodos tradicionales, el cambio no solo puede ser en la parte práctica, la cual ha cobrado relevancia, sino debe abordarse desde teorías que expliquen y permitan el desarrollo conceptual de los alumnos.

De acuerdo con la propuesta de Vygotsky (2006) existen dos tipos de conceptos, los cotidianos y científicos. Los primeros son adquiridos a partir de las experiencias concretas y de forma no intencionada; mientras que los conceptos científicos se desarrollan de forma consciente y tienden a hacer generalizaciones. Además un concepto científico hace referencia al conocimiento de los rasgos y propiedades esenciales de los diferentes objetos o fenómenos de la realidad objetiva, así como de las relaciones entre ellos. Estas propiedades tienen una jerarquía que permite identificar si un objeto de conocimiento pertenece o no a dicho concepto (TALIZINA, 2019).

Davídov (1988) y Galperin (2009) consideran que el principal objetivo de las matemáticas escolares es lograr la comprensión del concepto de número en los alumnos. Éste es concebido como el resultado de la relación entre una magnitud y la medida, el cual involucra la acción de medición. El trabajo con la medida permite introducir el concepto de unidad, es decir, de la relación de aquello que se mide y que es igual a su medida. Esta medida permite formar el sistema numérico decimal, considerando a la decena como una nueva medida, producto del aumento de la medida anterior (unidad) una cierta cantidad de veces. De manera similar se introducen otras medidas y clases (posicionales). 
La inclusión del sistema numérico decimal permite trabajar de inmediato con las operaciones de suma y resta, y sobre la base del concepto de la medida se inicia también la multiplicación. La multiplicación es obtenida al aumentar una medida una cierta cantidad de veces. La división permite obtener la primera medida (unidad), se conoce la medida y magnitud, pero se desconoce la cantidad de veces que fue utilizada (TALIZINA, 2019).

Después del trabajo de las acciones matemáticas, la solución de problemas matemáticos es posible platearla como una actividad intelectual (TSVETKOVA, 1999). En otras investigaciones (ROSAS; SOLOVIEVA, 2019) hemos enfatizado en diferenciar su forma de trabajo, debido a que es común percibir en las aulas escolares que los docentes solo plantean la solución de problemas como una tarea más, no se trabaja la reflexión del proceso de solución y queda de forma incompleta dicho proceso, solo se enfatiza el aspecto operativo. En nuestros trabajos previos (ROSAS; SOLOVIEVA; QUINTANAR, 2017; ROSAS; SOLOVIEVA, 2019) hemos identificado que los alumnos de primaria desarrollan el concepto de número, sistemas numéricos, acciones matemáticas, operaciones matemáticas y solución de problemas simples y complejos, es por ello que proponemos este contenido matemático como necesario en la escuela primaria.

\section{La formación del interés cognoscitivo durante las clases de matemáticas}

La caracterización del interés cognoscitivo y del contenido específico de las matemáticas desde la teoría de la actividad fueron la base para el análisis de dos grupos de tercer grado de primaria (colegios privados). El análisis principal de las experiencias en el aula escolar que presentamos en este estudio será a partir del enfoque cualitativo. Se trabajaron con dos grupos de tercer grado de primaria con condiciones semejantes en la cantidad de alumnos y en tener un método específico de enseñanza de las matemáticas. En un colegio se utilizaba el modelo Montessori y en otro un método basado en el modelo de Vygotski, ambos fueron denominados de esa forma por las maestras de los colegios correspondientes. Los colegios se ubican en la misma ciudad y son privados. Se utilizaron como instrumentos la observación de clases, la entrevista semi-estructurada para 
maestras y la evaluación dinámica para los alumnos. Nuestro primer objetivo fue caracterizar la forma de enseñanza, es decir, los contenidos, las tareas, las orientaciones y los tipos de colaboración que utilizan las maestras. Después nos propusimos identificar los conceptos y habilidades que los alumnos desarrollaban a partir del método de enseñanza de sus colegios.

\section{Caso colegio Montessori}

En este colegio existe una estructura diferente para impartir las clases. Los grupos son multigrado, aunque para nuestro caso se trabajó con alumnos de tercer grado, los cuales tomaban clases con alumnos de primero y segundo, además, recibían clases específicas para su grado. Los alumnos pueden tomar clases sentados en un tapete o trabajar en las mesas. También, en el ambiente (aula escolar) hay dos guías, una guía imparte tres días a la semana solo método Bancubi y método Montessori para la enseñanza de las matemáticas, y otra guía es la titular e imparte todas las asignaturas. Por esta razón, se aplicó la entrevista a las dos guías y se realizó una evaluación dinámica individual con ocho alumnos de tercer grado de primaria.

Durante la entrevista, las guías comentaron que la enseñanza de las matemáticas desde este enfoque era “muy sensorial”. La guía que utiliza el método bancubi y Montessori mencionó que el objetivo de su enseñanza es "el desarrollo de habilidades del pensamiento cuantitativo y lógico, por ejemplo, el razonamiento, la probabilidad, estadística y lógica” y conceptualiza el número como "un amigo/enemigo, son parte de la vida cotidiana, por ejemplo, el peso monetario". La maestra titular comentó que las matemáticas "se relacionan con la abstracción del cerebro en algo tangible, por ejemplo, observar la geometría del sol" y el número "tiene que ver con la historia". Para lograr sus objetivos de enseñanza consideran: la actitud, forma de presentar el material y su uso, el acompañamiento y corrección, así como la constante observación de la disposición (emocional) del alumno.

Durante las clases se percibió que la observación directa es el principal medio de construcción de conocimiento y verificación del mismo. Las guías 
constantemente realizan preguntas acerca de las características físicas de los objetos y sus relaciones cuantitativas. Describimos el siguiente ejemplo sobre la clase de fracciones equivalentes: la guía les pide a los alumnos construir con los cubos bancubi un edificio (horizontal) de 2 de ancho por 5 de largo y 1 de alto. Después la maestra les comenta que ese edificio es un entero, les pide que partan ese entero a la mitad. Una vez que los alumnos realizan la indicación, la maestra les comenta que eso se llama fracciones y les comentó que "eso que partieron son fracciones, hoy vamos a ver fracciones con equivalencias, valen lo mismo, lo cual puede ser dicho de diversas formas, yo me llamo Gabriela y me dicen Gaby, ¿cuántas maneras tienen de decirte?” La guía pregunta a cada alumno y un alumno respondió: “Felipe, Felis, Felipillo”, la maestra le preguntó: “¿eres el mismo?”, y el alumno respondió: "sí". Estas preguntas fueron realizadas con cada alumno. Posteriormente la guía propone dividir ese entero en diversas partes posibles hasta que llega a 3 y 4 partes. Los alumnos dicen que no es posible y la maestra les reitera "el entero que está formado por diez partes no se puede partir en 3 ni en 4 . Ahora partan en 5”. Después de la indicación anterior, la guía preguntó a un alumno “Cómo le vas a decir a ese cachito que tienes en la mano?”, el alumno no contestó y la maestra le dio una pista "usamos número ordinales". Otro alumno dijo "empieza con "c, no con s" (este alumno quería decir quinto). Otro alumno le dijo “quin...” y la maestra preguntó “¿cuántos quintos?, un quinto es igual a dos décimos”. Este alumno realizaba gestos de confusión, al parecer no logró observar lo que la maestra le decía, e incluso mencionó en voz baja que eran dos cubos.

Fue posible identificar que existen diversas preguntas durante las lecciones, siendo la observación la principal vía de análisis para responder. Los alumnos suelen preguntar sobre el uso del material, casi no preguntan sobre el contenido de la clase, más bien ellos manipulan su material y van realizando acciones como formar los cubos, separarlos, unirlos. En la clase de fracciones le preguntamos a un niño qué estaba haciendo y él respondió "estoy partiendo el entero", le preguntamos qué significaba eso, nos dijo "estoy separando los cubos en cachitos", insistimos en preguntar “¿en cuántos cachitos?” y nos contestó "ahora en... (mientras separaba cada uno de forma azarosa)”. 
Las guías buscan que todos los alumnos participen, las ayudas que les brindan a los alumnos normalmente son con las respuestas o con el inicio de la palabras. Las tareas que se plantean son de la siguiente forma: primero con el uso de material, después dibujan, y posteriormente escriben los números correspondientes. Además se observó que los alumnos pedían más tareas matemáticas, no les gustaba que terminara la clase de matemáticas. Por último, se percibió que los alumnos se relacionan de forma adecuada con sus guías y entre ellos. Cuando algún alumno solicita ayuda se le es brindada, aunque en lugar de explicarle se le termina respondiendo o se le describe el procedimiento de uso del material.

Las sesiones de evaluación dinámica con los alumnos se aplicaron de forma individual pero dentro del ambiente (aula escolar). Primeramente, fue posible percibir que ellos aún no aprendían la solución de algoritmos matemáticos, por lo que solo se aplicaron las tareas de preguntas sobre concepto y sistema numérico decimal y solución de problemas. Los alumnos en general mostraban una actitud positiva hacia las matemáticas, mencionaban que les gustaba mucho porque trabajaban con el material y la maestra era muy agradable. Solo una alumna mostró un poco de preocupación al realizar las tareas con nosotros, la guía nos explicó que fue porque ella tenía dificultades de aprendizaje en su anterior colegio.

El concepto de número que los alumnos han formado es de carácter absoluto, de acuerdo con Talizina (2019). Los alumnos perciben la relación cuantitativa del numero a partir de la recta numérica, es decir, 8 es mayor que 5 porque va después del 5 en la recta numérica. A pesar de identificar la medida y la cantidad, estas son percibidas de forma aislada e incluso los alumnos se centran más en la cantidad. Además, los alumnos requerían del apoyo visual y manipulación de su material, a pesar de tener la posibilidad de intentar resolver las tareas sin el material, la mayoría prefería utilizarlo y corroborar sus resultados. Ellos observaban constantemente su material, como si el material fuera a darles la respuesta o como si tuvieran que representar sus respuestas. Esta conducta fue muy interesante, en ocasiones lograban en otras no, y preferían el uso de sus dedos para contar. Los alumnos preguntaban a la 
investigadora sobre las tareas, pedían que se repitiera y ellos repetían de la misma forma, no cambiaban palabras. Esta repetición no les ayudaba, por lo que se les tuvo que explicar y demostrar mediante el dibujo concreto para que ellos identificaran acciones de aumento o conversión. Sus procedimientos eran desplegados, por ejemplo en lugar de utilizar la multiplicación ellos realizaban la suma con sus cubos. Por último, se intentó complejizar el tipo de apoyo utilizando el plano perceptivo esquemático (relación de una distancia entre tiempo) y solo dos alumnos lograron comprender esta relación mediante la multiplicación, es decir, a partir de la estimación de resultados " 9 x10 $=90,9$ x $2=18,90+18=98$, la respuesta es 12 " la forma en que justificaban su resultado fue a partir de la correcta solución del cálculo, no lograban explicar qué ocurría con el problema. Por ultimo, en la tarea de crear un problema para sus compañeros, los alumnos solo referían situaciones que implicaban un aumento o una disminución "Si tengo 1000 bombones y se comen 99 ¿Cuántos quedan?”

De forma general podemos identificar algunos elementos sobre el interés cognoscitivo durante las clases de matemáticas en este colegio. Primeramente, se percibe que a todos los alumnos les gustan las matemáticas. También fue posible identificar la constante curiosidad por el uso del material, los mismos alumnos comentan que el material les gusta mucho e incluso cuando están en clases ellos lo manipulan de diversas formas, y piden más actividades. Existe una conexión emocional de agrado hacia el material, aunque este no siempre es el mejor medio o tipo de apoyo para solucionar las tareas de matemáticas. Un alumno cuando intentaba resolver el problema que implicaba $98 \mathrm{~km}$ entre 8 horas, solo jugaba con los cubos porque no sabia de que forma utilizar el material, formaba con sus cubos 98 (nueve cubos verdes y ocho azules) y 8 (ocho cubos azules) pero intentaba juntar los azules, y los rojos quedaban del otro lado, después de varios intentos, lo invitamos a descubrir otros medios, se le explicó de forma perceptiva la relación matemática y logró con apoyo conjunto resolver, comprendió que tenía que distribuir esos $98 \mathrm{~km}$ en las 8 horas, y empezó con la estrategia de reparto por estimación.

Las características del interés cognoscitivo que se identifican en estas 
clases son: 1) el constante uso de preguntas que utilizan las guías, las cuales podrían pensarse como algo positivo que genere interés cognoscitivo, sin embargo, los alumnos solo responden lo que ellos ven, por lo que no hay una tensión cognitiva, ellos responden a atributos físicos de los objetos y su respuestas son correctas, pero al parecer ellos no identifican un problema que deben resolver, 2) podríamos inferir el esfuerzo por querer resolver las tareas mediante el uso del material, quizá para confirmar que ellos podían resolver. Cuando utilizamos las tareas complejas de evaluación, los alumnos solo repetían los enunciados y miraban el material como si el material les diera las respuestas o como si tuvieran que responder mediante el material, quizá ellos estaban recordando procedimientos previos que les ayudara pero no lograban buscar activamente otras formas de solución. Es posible también considerar que nuestras tareas no ocasionaron un interés inicial en los alumnos, sin embargo, es necesario comentar que cuando se les brindaba la explicación matemática y los alumnos resolvían, ellos expresaban agrado y un afán por saber, además se les daba otro ejercicio similar para saber si ellos habían comprendido, e incluso ellos daban otros ejemplos una vez que resolvían, se notaba que se mantenían a la expectativa, 3) las tareas se plantean para el trabajo individual pero entre ellos se preguntan sobre cómo van y se ayudan a dar solución si es necesario.

Por lo que, para estos alumnos existía mayor curiosidad y respuesta emotiva hacia los materiales y relación afectiva con los adultos, pero era posible mostrar una actitud activa (afán de saber) cuando se les explicaba las relaciones matemáticas, lo cual al parecer identificaban como algo novedoso y buscaban la forma de demostrar que si podían.

\section{Caso Colegio Vygotski}

Durante la entrevista, la maestra mencionó que las matemáticas son "una materia que se enseña en la escuela, qué involucra números y su aplicación está en la vida diaria”. Además, expresó que un concepto necesario de enseñar en la escuela primaria es el "concepto de número, el cual tiene como características una 
medida, magnitud y cantidad de veces", también refirió que es necesario enseñar "el sistema numérico", enfatizando en el valor posicional. Aunque la maestra mencionó que su método es basado en la teoría de Vysgotski, y por ello se denominó así este colegio, fue posible identificar que la maestra incluye en su discurso y en su planeación conceptos de la teoría de la actividad aplicada a la enseñanza.

Durante la observación de clase, también de fracciones con en el colegio anterior. La maestra introdujo este tema a partir de un repaso de las características del número y sus relaciones para lograr la acción de multiplicación. La maestra les recuerda el tema de la multiplicación "esta (multiplicación) es muy interesante porque ustedes ya saben que significa, que es el 5 y el 2 (señala una lámina con las tablas de multiplicación)" y pide a los alumnos que mencionen qué significa cada elemento: medida, magnitud y cantidad de veces. A dicha solicitud los alumnos contestan "medida por cantidad de veces y nos da la magnitud". A partir de esto se recupera como característica del número a la medida y la maestra pide ejemplos a los niños, quienes dicen que pueden usar como medida la "regla, lápiz, goma, libreta, estuche, la unidad" y de magnitud, lo que pueden medir (largo de ventana, pizarrón, largo del pizarrón). Posteriormente, la maestra les da una tira de papel del mismo tamaño a todos y les indica que midan un lado del cuaderno, antes de esto resume los elementos mencionados (medida, magnitud, cantidad de veces). Después la maestra pega unas láminas (papel bond) con la información trabajada y con la tabla de registro de los ejercicios que están realizando. En seguida la maestra les da una medida (tira de listón) más grande que la portada de su cuaderno, aquí introduce el tema de "fracción", señala sus componentes matemáticos y un niño dibuja en la tabla de registro la mitad de un cuadrito (unidad).

Posteriormente la maestra muestra una hoja completa y la llama "unidad" y pega abajo dos mitades de hoja, debajo de esto coloca cuatro cuartos de hoja (manteniendo la unidad) y debajo de estos ocho octavos de hoja, la maestra enfatiza durante esta acción cuántos cuartos se necesitan para juntar la unidad, cada pedazo tiene su número correspondiente. Después la maestra reparte el material a los alumnos, el cual ellos habían elaborado previamente, una hoja la utilizan como unidad, cuatro partes de esa unidad como cuartos y ocho como octavos; cada 
parte tiene su número correspondiente. Posteriormente, la maestra les da a los niños una hoja con cinco situaciones problemáticas, y ellos realizan el primero de forma conjunta, enfatizando que primero colocan las medidas (materializadaspedazos de papel) que les indica (unidades, cuartos, octavos). La siguiente actividad fue una hoja con ejercicios de fracciones, en esta ocasión los niños no utilizaban su material de fracciones sino dibujaban el resultado, a partir de un entero (unidad) dividían y señalaban el resultado iluminando la parte solicitada, finalmente escribían el número correspondiente. Este ejercicio fue individual y se realizó sin errores. Para finalizar el tema de fracciones, la maestra hizo un resumen de lo visto, los alumnos participaban dando información y ejemplos. Se observó que los alumnos se preguntaban entre ellos sobre los resultados, y se pedían ayuda cuando no comprendían. La ayuda que se brindaban era muy similar a la explicación que daba la maestra. Una alumna ayudó a su compañero diciendo los pasos: - Alumna: primero ¿qué debes hacer? -Alumno: leer el enunciado, Alumna: ¿qué te dice el enunciado?, -Alumno: Que tengo dos cuartos de litros de jugo, y tres octavos de litros de agua... - Alumna: ¿cuál es tu medida? ¿Cuál es tu unidad? Coloca aquí una medida y acá otra, completa la unidad... Así continuaban dialogando hasta que resolvían la tarea.

El alumno resolvió de forma individual los demás ejercicios. Por último, todos corroboran sus resultados en grupo. La maestra para verificar el proceso de solución les pide que verifiquen porque no cree que sea la respuesta correcta, entonces un alumno tomó el material y explicó a la maestra el procedimiento. La maestra sonrió y comentó que era una trampa.

La evaluación dinámica aplicada a los alumnos evidenció que los alumnos identificaban las características del concepto de número y se orientaban a partir de ellas para solucionar diversas tareas, así como el uso de algoritmos matemáticos para solucionar problemas matemáticos. Sin embargo, existieron errores en el cálculo y para mantener la medida, debido a la impulsividad de querer responder rápido. Estos errores fueron superados por los alumnos, en ocasiones de forma independiente y en otras requerían apoyo verbal escrito. Los tipos de ayuda con estos alumnos fueron mínimos, se observaba que ellos repetían de forma activa la 
información necesaria para resolver las tareas, por ejemplo "me dice que un tren recorre $98 \mathrm{~km}$, la medida es $\mathrm{km}$, y tarda 8 horas, la medida es horas, me pregunta ¿cuántos km hace en una hora?, si tengo las medidas y el total del recorrido, ¿sería mi magnitud 98?” Este alumno resolvió con el algoritmo de la división pero expresó la relación nuevamente entre una Magnitud y una medida, el valor que tenía que encontrar era la cantidad de veces, aunque el alumno no logró expresarlo en voz alta, logró comprender la relación matemática y lógica del problema. Por último, en la tarea de crear un problema para los compañeros, los alumnos mostraron agrado hacia esta tarea, de hecho todos mencionaron que escribirían problemas difíciles y dos alumnos dijeron que serían problemas sin solución, que les pondrían una trampa a sus compañeros.

A partir de la descripción anterior el interés cognoscitivo se percibe de la siguiente forma: 1) en la enseñanza, la maestra utiliza también preguntas, las cuáles involucran la identificación de las relaciones entre las características del concepto de número, además, las preguntas también son utilizadas por los alumnos como análisis del procedimiento para resolver tareas, 2) las tareas propuestas incluyen objetivos intelectuales, lo que lleva a los alumnos a reflexionar sobre información específica y necesaria para la solución, por ejemplo, cuando están resolviendo problemas matemáticos repiten activamente la pregunta del problema y la situación del problema, y no solo resuelven la operación matemática, 3) variedad del contenido de las tareas, a pesar de haber observado el tema de fracciones fue posible identificar que se proponen a partir de diversas condiciones, no solo objetos concretos, sino con medidas diversas (longitud, volumen, peso), e incluso en el material para resolver las tareas, 4) en la enseñanza también se observa que se explican los procedimientos generales para llevar al éxito a los alumnos, los cuales se organizan en tarjetas de orientación, 5) la solución de tareas en equipo, los alumnos se proponen como objetivo dar respuesta a las tareas, para lo que dialogan entre ellos, preguntan a la maestra y finalmente muestran agrado entre ellos cuando logran dicho objetivo. De esta forma, es posible percibir que el interés cognoscitivo está presente en este tipo de método de enseñanza y durante las clases que la maestra imparte. Aunque, también fue posible identificar la 
curiosidad, el afán de saber y el interés cognoscitivo durante las clases y en la evaluación realizada con los alumnos.

En relación con las preguntas que dirigieron este trabajo podemos comentar que el interés cognoscitivo puede formarse cuando en el método de enseñanza se incluyen los conceptos matemáticos y no solo definiciones, además, los alumnos necesitan resolver las tareas en las que deben utilizar esos conceptos científicos (relación entre la actividad intelectual y práctica), en el caso de las matemáticas, el concepto de número fue identificado.

Si bien, en ambos colegios se identifica el uso de materiales, diversos planos de acción y el trabajo colectivo, es posible percibir que la diferencia principal está en el contenido matemático. En el caso del colegio Montessori fue posible identificar la enseñanza de la cantidad y la medida de forma aislada, así como el establecimiento de tareas que favorecen a la curiosidad, mientras que en el colegio Vygotski es posible percibir el concepto de número y la solución de problemas intelectuales. Lo cual fue visto en el análisis de la evaluación dinámica con los alumnos, cuando los alumnos del colegio Montessori recibían la explicación matemática, la relación entre la medida con la cantidad de veces, los alumnos resolvían y se esforzaban por continuar con las tareas, manteniéndose a la expectativa. Aunque, los alumnos continuaban dependiendo del material, y esto engancha al pensamiento a la experiencia sensorial. Es posible suponer que más adelante quizá los alumnos desarrollen el interés cognoscitivo.

Un aspecto positivo de estos métodos es que los alumnos refieren el agrado por las matemáticas, por sus maestras y por el material. Además de que mantienen una situación emocional positiva, las maestras y alumnos no se preocupaban por las calificaciones o por evitar algún tipo de sanción. Estos colegios tienen una área de pedagogía que se encarga de profesionalizar a las alumnas y brindar una guía. Podemos inferir que este acompañamiento con las maestras les favorece para lograr los objetivos establecidos por sus métodos de enseñanza y repercute en su estado emocional. 


\section{Conclusión}

La propuesta de análisis descriptivo del interés cognoscitivo desde la perspectiva del enfoque histórico-cultural realizado en las clases de matemáticas nos permite señalar que la enseñanza puede adoptar el objetivo dirigido hacia el desarrollo tanto de la actividad intelectual como de la personalidad. En el caso de las matemáticas, el concepto de número favorece el desarrollo del pensamiento pero también de la actitud creativa. Por último, consideramos que el método de la maestra del colegio Vygotski es un ejemplo de la amplia posibilidad que tiene el maestro para organizar el desarrollo de los alumnos de forma integral. Este tipo de experiencias pueden incluirse para la propuesta de modelo educativos y para la formación de profesores.

\section{Referencias bibliográficas}

AREVALO, E. ¿Cómo enseñan las matemáticas en la escuela primaria? XIV Conferencia Interamericana de Educación Matemática. Tuxtla Gutiérrez, Chiapas, México, 2015.

BOZHOVICH, L. I. La personalidad y su formación en la edad infantil. La Habana: Editorial Pueblo y Educación, 1976.

BOZHOVICH, L. I. Las etapas de formación de la personalidad en la ontogénesis. En: DAVIDOV, V; SHUARE, M (Org.). La psicología evolutiva y pedagógica de la URSS. Antología. Moscú: Editorial Progreso, 1987.

DAVIDOV, A.; MARKOVA, A. El desarrollo del pensamiento en la edad escolar. En: DAVIDOV, V; SHUARE, M (Org.). La psicología evolutiva y pedagógica de la URSS. Antología. Moscú: Editorial Progreso, 1987.

DAVIDOV, V.V. La enseñanza escolar y el desarrollo psíquico. Investigación psicológica teórica y experimental. Moscú: Editorial Progreso, 1988.

DOMINGUEZ, L. El desarrollo psicológico humano como proceso de continuidad y ruptura: "la situación social del desarrollo", Educação y filosofía Uberlândia, 29 (57), pp. 21-42, 2015. DOI: https://doi.org/10.14393/REVEDFIL.issn.01026801.v29n57a2015-p21a42.

ELKONIN, D. Hacia el problema de periodización del desarrollo en la edad infantil. En QUINTANAR, L; SOLOVIEVA, Y (Org.). Las funciones psicológicas en el desarrollo del niño. México: Trillas, 2011. 
GALPERIN, P. Ya. La formación de los conceptos y de las acciones mentales. En QUINTANAR, L.; SOLOVIEVA, Y. (Comps.). Las funciones psicológicas en el desarrollo del niño. México: Trillas, 2009.

PADILLA, J.; MEJÍA, M. Factores motivacionales y su relación con el rendimiento académico en alumnos en el nivel primaria. Revista de estudios clínicos e investigación Psicológica, 10 (20), pp. 248- 257, 2020.

ROSAS, Y. Análisis de los métodos de enseñanza de las matemáticas desde la teoría de la actividad. Tesis para obtener el grado de Doctor en Educación, Universidad Iberoamericana de Puebla, 2019.

ROSAS, Y.; SOLOVIEVA, Y. Trabajo con solución de problemas matemáticos en tercer grado de primaria: análisis en dos escuelas privadas. Ensino em revista, 26 (2), pp. 415-436, 2019. DOI: https://doi.org/10.14393/ER-v26n2a2019-6.

ROSAS, Y., SOLOVIEVA, Y.; QUINTANAR, L. La formación de concepto de numero: aplicación de una metódica en una institución mexicana. En: TALIZINA, N.; SOLOVIEVA, Y.; QUINTANAR, L. Enseñanza de las matemáticas desde la teoría de la actividad. México: CEIDE, pp.87-105, 2017.

SANCHÉZ, M.; DOPICO, H. El interés como fuerza motivacional, una metodología para el control en la educación física. Revista digital Argentina, 11 (98), 2006.

SECRETARIA DE EDUCACIÓN PÚBLICA. Aprendizaje clave para la educación integral. México: SEP, 2017.

SOLOVIVEA, Y., GONZALEZ-MORALES, C., ROSAS, Y., MATA, A.;

MORALES, A. Resultados de investigación educativa desde el modelo histórico cultural y la teoría de la actividad la Universidad Iberoamericana de Puebla.

Ensino em revista, 27, pp. 1256-1274, 2020. DOI: https://doi.org/10.14393/ERv27nEa2020-4.

SHÚKINA, G. I. Los intereses cognoscitivos en los escolares. Ciudad de la Habana, Editorial Libros para la Educación, 1978.

TALIZINA, FN. La teoría de la actividad aplicada a la enseñanza. México: Benemérita Universidad Autónoma de Puebla, 2019.

TALIZINA, N., SOLOVIEVA, Y.; QUINTANAR, L. La aproximación de la actividad en Psicología y su relación con el enfoque histórico-cultural de L. S. Vygotsky. Novedades educativas, 230, p. 4-8, 2010.

TSVETKOVA, L. (1999). Neuropsicología del intelecto. La Habana: UAEM.

VYGOTSKI, L. El desarrollo de los procesos psicológico superiores. Barcelona: Crítica, 2006. 
VYGOTSKI, L. Obras escogidas. Tomo 3. Madrid: Visor, 1995.

VYGOTSKI, L. Aprendizaje y desarrollo intelectual en la edad escolar. Infancia y aprendizaje, 27-28, pp. 105-116, 1984. DOI:

https://doi.org/10.1080/02103702.1984.10822045.

WEISS, E., BLOCK, D., CIVERA, A., DÁVALOS, A.; NARANJO, G. La enseñanza de distintas asignaturas en escuelas primarias: una mirada a las prácticas docentes. Revista Mexicana de Investigación Educativa, 24 (81), pp. 349-374.

Recebido em setembro de 2020. Aprovado em dezembro de 2020. 